Article

\title{
The Social-Ecological Keystone Concept: A Quantifiable Metaphor for Understanding the Structure, Function, and Resilience of a Biocultural System
}

\author{
Kawika B. Winter ${ }^{1,2,3, *(\mathbb{D})}$, Noa Kekuewa Lincoln ${ }^{4}\left[\right.$ and Fikret Berkes ${ }^{5}$ (D) \\ 1 Hawai'i Institute of Marine Biology, University of Hawai'i at Mānoa, Kāne'ohe, HI 96744, USA \\ 2 Department of Natural Resources and Environmental Management, University of Hawai'i at Mānoa, \\ Honolulu, HI 96822, USA \\ 3 National Tropical Botanical Garden, Kalāheo, HI 96741, USA \\ 4 Department of Tropical Plant and Soil Sciences, University of Hawai'i at Mānoa, Honolulu, HI 96822, USA; \\ nlincoln@hawaii.edu \\ 5 Natural Resources Institute, University of Manitoba, Winnipeg, MB R3T 2N2, Canada; \\ Fikret.Berkes@umanitoba.ca \\ * Correspondence: kawikaw@hawaii.edu
}

Received: 31 July 2018; Accepted: 11 September 2018; Published: 14 September 2018

check for updates

\begin{abstract}
Social-ecological system theory draws upon concepts established within the discipline of ecology, and applies them to a more holistic view of a human-in-nature system. We incorporated the keystone concept into social-ecological system theory, and used the quantum co-evolution unit (QCU) to quantify biocultural elements as either keystone components or redundant components of social-ecological systems. This is done by identifying specific elements of biocultural diversity, and then determining dominance within biocultural functional groups. The "Hawaiian social-ecological system" was selected as the model of study to test this concept because it has been recognized as a model of human biocomplexity and social-ecological systems. Based on both quantified and qualified assessments, the conclusions of this research support the notion that taro cultivation is a keystone component of the Hawaiian social-ecological system. It further indicates that sweet potato cultivation was a successional social-ecological keystone in regions too arid to sustain large-scale taro cultivation, and thus facilitated the existence of an "alternative regime state" in the same social-ecological system. Such conclusions suggest that these biocultural practices should be a focal point of biocultural restoration efforts in the 21st century, many of which aim to restore cultural landscapes.
\end{abstract}

Keywords: alternative regime state; portable biocultural toolkit; social-ecological system theory; Hawaii; Colocasia esculenta

\section{Introduction}

\subsection{Social-Ecological Systems and the Application of Ecological Terminology}

This paper emphasizes the concept that humans are a part of-not separate from-nature [1], supporting views established by Berkes and Folke [2], and Berkes et al. [3], which hold that the delineation between social systems and natural systems is arbitrary and artificial. Several frameworks for understanding such social-ecological systems have been put forth (e.g., [4-6]), but some have pointed out a disconnect between the frameworks-proposed to understand social-ecological systems - and the biocultural elements that are at the foundation of such systems [7]. This research aims to bridge that gap by (a) presenting theories and methods associated with quantifying biocultural 
relationships within social-ecological systems; (b) demonstrating how restoring the function of "keystone" components is essential to restoring the structure of social-ecological systems that are observed to be in decline; and (c) demonstrating how restoring the function of "redundant" components is essential to restoring the resilience of such systems. As with our other publications on social-ecological systems, we follow the Walker et al. definition of resilience as the capacity of a system to absorb disturbance and reorganize while undergoing change so as to still retain essentially the same function, structure, identity, and feedbacks [8].

Following Berkes [9], we use the terms "ecological subsystem" and "social subsystem" when discussing particular sides of the social-ecological spectrum, and will do so even when the referenced text uses the term "ecosystem". This allows us to discuss each of the two sides, while maintaining that the two subsystems are not autonomous from one another. We follow others in applying common ecological terms to set up a logical framework for understanding social-ecological systems [10-13]. This paper uses accepted terminology such as 'function', 'functional group', 'diversity', 'keystone', 'redundant', 'regime shift', and 'stable state' of Anderies and Janssen [14] in discussing social-ecological systems. This paper uses the term, 'alternative regime state', to describe alternative stable states that can exist within the same social-ecological system, such as flooded-field agriculture versus rain-fed agriculture practiced by the same culture. An 'alternative regime state' describes different stable states that can exist within the context of the same social-ecological system, whereas a 'regime shift' indicates a stable state that exists within a different social-ecological system altogether, such as a rural agricultural community being transformed into a city and inducing a concurrent shift in the dominant culture. This notion is explored in more detail below.

\subsection{Quantifying Biocultural Elements within Social-Ecological Systems}

Theories relating to co-evolutionary relationships between human and natural systems are not new [15]. In the 5th century BC, the Greek philosopher Herodotus voiced his observation that events shape both people and nature, and that people and nature interact and evolve together through these events. More recently, Winter and McClatchey [16,17] put forth theories to quantify these co-evolutionary relationships, and established methods for measuring fundamental units of interaction between people and plants-or linked biological-sociocultural relationships (henceforth referred to as biocultural relationships) - in a way that is scalable from simple interactions (one person and one plant) to complex relationships (all of humanity and all plants). Such an approach has been used to address hypotheses about the evolution of interactive relationships [16-18].

The Quantum Co-evolution Unit—or QCU—(Figure 1) is a unit to measure linked, co-evolving relationships such as those observed in social-ecological systems [16-18]. These relationships will henceforth be referred to as "biocultural elements" of such systems. A set of QCUs within a social-ecological system can be quantified (Figure 2, [17]) and assessments of these populations at different times can demonstrate co-evolving biocultural relationships [16,17]. As in many disciplines, units can be considered at different scales for both the ecological component (ecosystem, genus, species, etc.) and the social component (socio-cultural system, community, individual, etc.), to assess the health of diffent aspects of a social-ecological system. The research presented here contends that QCUs can be used as a unit to quantify biocultural elements, such as the following:

- exploring the concept of functional groups within social-ecological systems,

- quantitatively classifying particular elements as either keystone components or redundant components of social-ecological systems,

- quantitatively relating loss of keystone components to loss of social-ecological system structure and function,

- quantitatively classifying loss of redundant components to diminished resilience in social-ecological systems,

- identifying alternative regime states within a single social-ecological system,

- quantifying regime shifts between social-ecological systems. 
Possessing such an understanding can be key to informing biocultural restoration efforts.

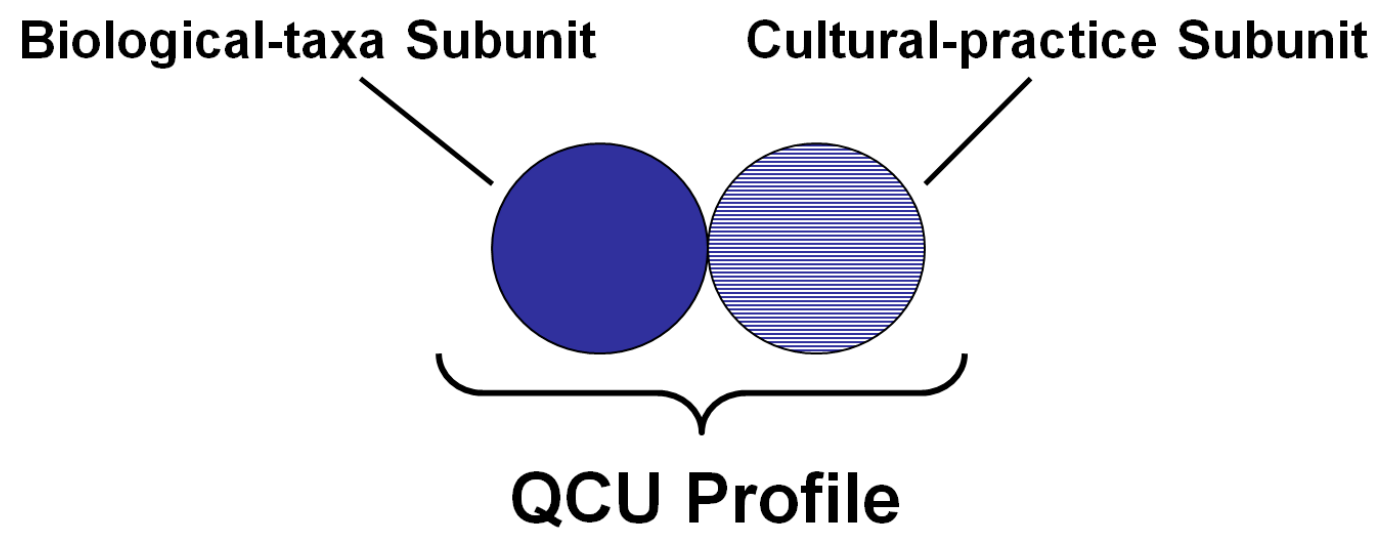

Figure 1. The Quantum Co-evolution Unit (QCU), which relates to the co-evolutionary relationship between biological taxa and human cultures. It is composed of two subunits-the biological-taxa subunit, and the cultural-practice subunit-and is used as a metric for biocultural diversity. The complete unit of the QCU is referred to and described by its QCU profile [17].
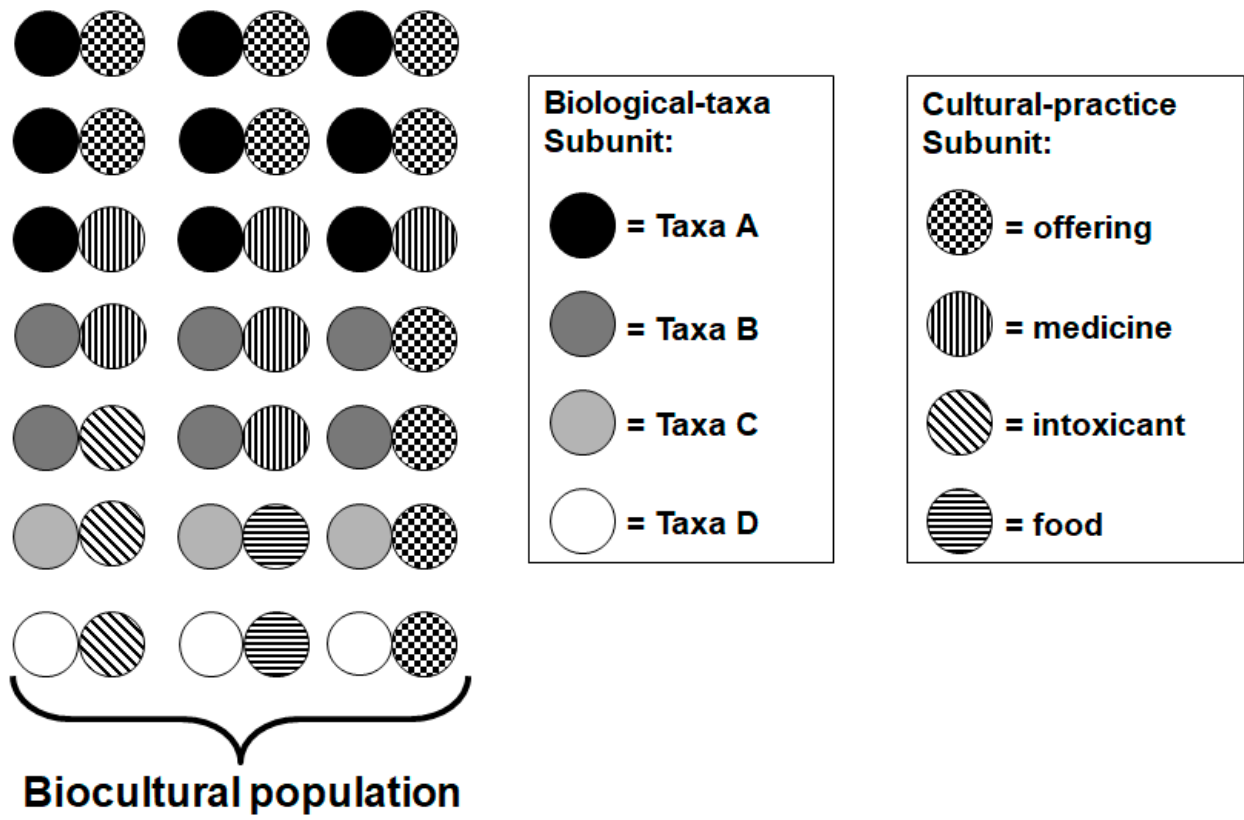

Figure 2. A QCU population. A hypothetical collection of Quantum Co-evolution Units (QCUs) represented, within a social-ecological system, showing proportionality and frequency of various QCUs in relation to one another. The QCU population of a social-ecological system could be sampled over various points in time. Changes could be observed and further quantified. Such changes could include the adoption of new QCUs into the profile, deletion of QCUs from the population, and changes in individual QCU frequency within the population [17].

\subsection{The Hawaiian Social-Ecological System}

The evolution of social-ecological systems in Hawai' $i$ is uniquely understandable, in part, because the relatively late human colonization allows for tracing the entirety of human history. As such, Hawai'i has been described as a model for the study of social-ecological systems $[17,19]$.

Archaeological evidence indicates settlement no later than 1000 years ago [20], although use of oral history sources indicates initial voyages to the Hawaiian islands may have happened centuries prior [21-24]. In the pre-contact era-prior to contact with Europeans in 1778-the social-ecological system in Hawai'i was intensively managed to maximize resource abundance by attaining a stable state known in Hawaiian as "äina momona". 'Aina momona is descriptive of a stable state that can exist in alternative 
forms in Hawai'i, and is associated with both flooded-field and rain-fed agriculture [25]. This is a stable state that was brought about via a regime shift (originally from an ecosystem into a social-ecological system that maximized ecosystem services). The social-ecological system in the Hawaiian archipelago associated with the pre-contact era will henceforth be referred to as the "Hawaiian social-ecological system", which is identified by the similarity of linked human-ecological units-being the foundational culture, the available plant species, and the cultural uses of those plants—-that existed in the Hawaiian ecoregion in the pre-contact era.

Alternative states of 'aina momona existed in the Hawaiian social-ecological system, and while they had similar functional components with one another, they differed in structure across different regions of the archipelago. As has been described for ecological subsystems [26], it appears that the structures of these alternative regime states were shaped around different keystone components of the same system. Observations about differing structures of alternative regime states associated with either flooded-field or rain-fed agriculture [27] may be related to this phenomenon [28].

The structure of the Hawaiian social-ecological system has been in decline since the 19th century [29]. Gaining an understanding of the biocultural elements, and identifying them as either keystone or redundant components could be beneficial to efforts aimed at restoring the structure, function, and resilience of that system. We utilize theoretical foundations and logical assumptions to explore the the relative importance of biocultural elements in Hawaiian agricultural traditions-a core foundation within the pre-contact social-ecological system in Hawai'i.

\section{Theoretical Foundations}

\subsection{The Keystone Concept as Relates to System Structure and Function}

Paine [30] first described ecological 'keystone species', as occurring in a situation where patterns of distribution and density of species within an ecological subsystem are disproportionately affected by the activities of a single species. It has since become a major concept within the discipline, but has fueled decades of debate on definitions [31]. Ultimately, this debate stems from disagreements about how to quantify a metaphor. Is the concept of 'keystone' a biological reality, or is it a simple metaphor to understand a complex system? This paper argues the former, and following systems theory [32], asserts that the keystone concept holds true within social-ecological systems. This paper further asserts that it is possible to quantitatively determine a keystone component by assessing functional groups of biocultural elements within a system, and then analyzing the associated diversity within those functional groups.

The disproportionate influence of keystone species suggests there is no functional redundant within the system (Figure 3). If the keystone is removed, then a relatively large number of secondary extinctions would occur [33,34] and the system would reorganize itself with a different structure and function. This process is referred to as a regime shift [35]. Thus, keystone components play a major role in the structure and function of systems.

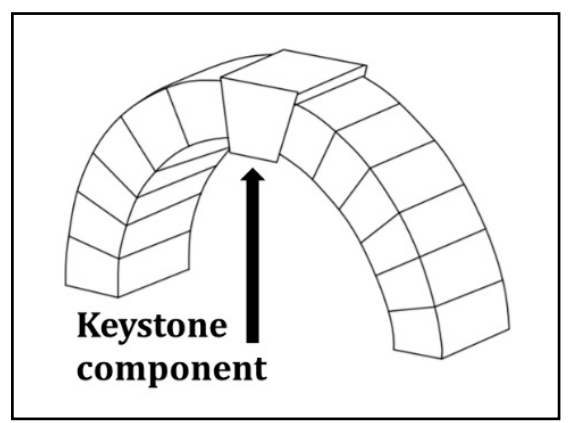

Figure 3. The keystone metaphor. The keystone is the component of a structure that is irreplaceable. Without the keystone, a structure could be reassembled, but could never be the same as if the keystone were present and functioning in its role. 


\subsection{The Social-Ecological Keystone Concept}

The keystone concept of has been applied to various cultural interactions with the biological world [10-12]. Garibaldi and Turner [11] define cultural keystone species as "the culturally salient species that shape in a major way the cultural identity of a people". Cultural keystone practices, described by Brosi et al. [12], are traditions that are so intrinsic to the culture that, if they were to disappear, the culture would be irreversibly altered. Thus, the usage of the keystone concept by researchers working within the social-ecological system paradigm is not new. However, this paper contends that a "social-ecological keystone" is actually a specific biocultural element (i.e., relationship); further, that social-ecological keystones can be quantitatively determined utilizing the theoretical concepts previously established by Winter and McClatchey $[16,17]$.

A relevant concept here is 'functional group'. In ecological subsystems, functional groups are used to lump together species with similar roles-such as top predator, generalist pollinator, nitrogen fixer, and so on. In social-ecological systems, members of functional groups would be biocultural relationships-such as applying herbal medicine, imbibing fermented sugars, weaving baskets, farming complex carbohydrates, and so on. This paper, therefore, refers to "biocultural functional groups", and has identified the QCU as a unit of measure for the biocultural elements within them.

Based in part on Davic's [31] definition of keystone species, this paper submits that a social-ecological keystone is a strongly interacting biocultural element within its functional group, whose top-down effect on biocultural diversity is large relative to all elements within the system. Biocultural elements, and therefore social-ecological keystones, are neither individual taxa nor individual practices, but rather the linked taxa-practice unit. If a social-ecological keystone is severely disabled (or goes extinct), then there would be no substitute without seriously compromising the structure and function of the system-possibly inducing a regime shift. Correspondingly, if a social-ecological keystone were to go extinct it would cause a cascading effect of secondary extinctions of biocultural elements, and subsequently affect the structure of both the ecological and social subsystems. The theories and methods explored in this manuscript could be used to document and understand such processes.

\subsection{The Influence of Crop Diversity and Cropping Systems on the Structure of the Hawaiian Social-Ecological System}

Functional groups associated with agriculture often determine the structure and function of social-ecological systems managed by agrarian societies because agriculture often dictates the form and hierarchy of the social subsystem, and is the foundation of the economy and politics within the social subsystem [36], which subsequently influences the management of the ecological subsystem. The central role of agriculture in cultural development, political complexity, material economy, and social norms of the Hawaiian social-ecological system has been well explored (see Lincoln and Vitousek [37] for a broad overview and detailed reference list). Based on these concepts, this paper holds the assumption that agriculture is a key biocultural functional group within social-ecological systems managed by agrarian societies.

Hawaiian agriculture manifested in highly diverse forms in the Hawaiian social-ecological system. The most salient division often used in anthropological discussion is the difference between wet (flooded-field, irrigated) and dry (rain-fed) agriculture [38,39]. The state environmental factors that drove the opportunities and constraints of agricultural development are highly organized, but not evenly distributed, in the Hawaiian archipelago. The distribution of geological age and rainfall, which subsequently drive soil fertility and land topography, created a spectrum of agricultural opportunties that spanned almost exclusively rain-fed opportunities on the young island of Hawai'i, to almost exclusively flooded-field opporunities on the oldest of the high islands, Kaua'i [27]. These agricultural forms had different requirements (e.g., levels of organization, infrastructural investment) and offered different effects (e.g., levels of resilience, economic surplus).

In brief, flooded-field agriculture was investment intensive, but low maintainenance while offering low vulnerability to both natural and social perturbances. Consequently, flooded-field 
agriculture supported socio-political systems on Kaua'i with more diversified social roles and stronger political stability. Conversely, the more labor intensive and vulnerable dryland agricultural systems of Hawai'i Island manifested socio-political systems that were more volatile, saw frequent regime shifts between political leaders, and spawned predatory political ambitions [28]. This implies the existence of "alternative regime states" within the same social-ecological system-a more resilient one built around flooded-field systems of agriculture, and a more vulnerable one built around rain-fed systems of agriculture. These alternative regime states existed even though they are based on the same biocultural elements in the same social-ecological system. The manifestations of the economy and the political systems differed due, at least in part, to the relative and absolute areas of the agricultural systems.

\subsection{Social-Ecological System Resilience and the Role of Redundant Components}

Resilience $[40,41]$ is a measure of a system's relative ability to absorb disturbance without changing into a different state (i.e., regime shift), such as a different biological community with different ecosystem services [35]. Biological diversity has been shown to be a key factor in the resilience of ecological subsystems $[8,42]$ because it helps to maintain desired states of dynamic regimes in the face of uncertainty and surprise, and also plays a major role in renewing and reorganizing those systems after disturbance [35]. Maintaining such desired states of dynamic regimes (i.e., "stable states") is more specifically dependent on "response diversity", which is the diversity of responses to environmental change among species that contribute to the same function in the system [43]. Therefore, the more nuanced value of diversity is the increased redundancy within functional groups [44]. Such 'functional diversity' refers to the number of species that perform the same function; if a member of a functional group were to be lost (either temporarily or permanently) via a disturbance event, then its function could be replaced by another species. Ecological systems with high response diversity increase the likelihood for reorganization and renewal into a desired state after disturbance [43]; and are, therefore, more resilient. If a set of functionally redundant species does not exhibit any response diversity, then they do not contribute to system resilience [35]. A loss of biodiversity-more importantly a loss of functional diversity and response diversity-is a major contributing factor in regime shifts $[35,45]$. In the context of ecological subsystems, regime shifts imply a shift in services which that subsystem provides to the socio-cultural subsystem, and are largely irreversible [35].

Throughout this paper we use the term, 'redundant component' to classify a biocultural element of a functional group that could be substituted if removed - that is, a component that is not a keystone. In accordance with systems theory [32], maintaining resilience in social-ecological systems relies on the management of biocultural diversity, including the seemingly redundant components of these systems. Such "social-ecological redundants" contrary to keystones, may not contribute significantly to the structure and function of social-ecological systems individually, however, if they represent response diversity, then such components may contribute significantly to the resilience of social-ecological systems.

We use the QCU (Figure 1) as a unit of measure to quantitatively classify redundant components of social-ecological systems within biocultural functional groups. This paper contends that biocultural functional redundancy exists in instances where a subunit of the QCU (either biological taxa or sociocultural practice) lost via a disturbance or other event can be easily transferred to other corresponding subunits which would ensure the persistence of that biocultural functional group. For example, using the biocultural functional group of "weaving a plant-based fiber", a culture may have five taxa which it uses to weave. If one of the five taxa were to go extinct, the sociocultural practice could continue because of the functional redundancy that exists in that biocultural functional group.

\subsection{Theoretical Assumptions}

This manuscript builds off of four theoretical assumptions in regards to the keystone concept:

1. Keystone function of a system can be viewed in terms of a functional group.

2. Keystone components of functional groups are dominants within that functional group. 
3. Dominant components of a functional group (i.e., keystone components of a system) are not necessarily dominant components within the overall system.

4. Shifting dominance within a keystone functional group replaces the keystone component of the system, thus influencing the structure of that system.

\section{Testing the Keystone Theory in Social-Ecological Systems}

\section{The Hawaiian Social-Ecological System as a Model}

There are countless social-ecological systems that could be chosen from around the world to model the theories explored in this paper, but in order to test the conceptual validity of these theories a model social-ecological system is ideal. High islands are excellent examples to discuss system function becuase they are big enough to possess all the biological, ecological, chemical, and physical processes needed for complete system study, yet small enough that the complexity of such systems is perceivable $[46,47]$. Kirch [19] outlines how both the social and ecological factors of Hawai'i, in particular, lend themselves to serving as a model system for biocultural understanding. State factors influencing ecology are either held constant (e.g., parent material, biota) or are extremely broad yet well organized (e.g., climate, age, topography). Simultaneously, social factors lend themselves to study due to the short timeframe of human colonization, the extreme isolation, and the high level of socio-political complexity achieved.

To exemplify the theories expressed above, we focus on the Hawaiian social-ecological system. At the point of contact with Europeans in 1778, this highly modified system was being managed to maintain a human population on the order of 300,000-800,000 people [48,49]. Furthermore, anthropological discussion has argued that Hawai' $i$ developed high levels of socio-political heirarchy manifested in complex systems of land tenure, resource mangement, and taxation, describing Hawai'i as one of nine civilizations to have independently developed into a state system [50]. The Hawaiian archipelago, therefore, represents an island-bound and intensively managed social-ecological system, with a population size and social structure that makes it comparable to the contemporary period.

\section{Methodology}

As this is an examination of the structure and function of a system which existed in the past, historical records and archaeological evidence were used to supplement actual observations. The methods described below were used to quantify biocultural diversity, identify key functional groups within systems, as well as quantitatively classify keystone components and redundant components that constitute these functional groups.

\subsection{Quantification of Biocultural Diversity}

This research considers a unit of biocultural diversity - referred to as the Quantum Co-evolutionary Unit (QCU) — as any one of the human needs, as described by Max-Neef et al. [51,52], which has a satisfier that comes from within the realm of biodiversity. QCUs were generally assessed from the standpoint of viewing them as components within functional groups that are embedded in the entire system, rather than as individual components standing alone within the context of the entire system.

\subsection{Assessing Biocultural Functional Groups in Social-Ecological Systems}

It is the assumption of this paper that within an agrarian society-such as that associated with the Hawaiian social-ecological system-social-ecological keystones can found within the biocultural functional groups associated with agriculture. Biocultural functional groups were identified by reviewing the seminal literature on ancient agricultural and associated practices [53-59]. From this literature review, commonly occurring categories of agricultural function clearly stood out and were identified to be used to define the biocultural fuctional groups. This literature, while select, forms the broad basis for vast majority of subsequent publications in traditional Hawaiian agriculture. 
Once the biocultural functional groups associated with agriculture were determined, their components were then quantified to classify them as a keystone component or a redundant component within each respective functional group. As we attempt to examine a time period in the past, data from historical records and publications were used. Handy et al. [54] is a comprehensively researched tome on ancient Hawaiian agriculture that was produced by the B.P. Bishop Museum in collaboration with anthropologists and a highly-respected, well-published, native-speaking Hawaiian ethnographer; it is widely considered the authoritative volume regarding Hawaiian agriculture. For the purposes of this research, the number of written lines dedicated to each biocultural relationship was used as a proxy for the relative importance of that element (see Table S1). Other sources of knowledge in this area exist [53-59], but those works were not systematically approached from the standpoint of plant-based biocultural relationships as was the work of Handy et al. [54]. The number of lines, therefore, provides the numerical quantification of each element subsequently used to calculate the indexes as described below. While this is an ad hoc approach utilizing only a single, albeit substantial, volume on Hawaiian agriculture, we employ this method to demonstrate the application of the QCU concept in a meaningful way and to provide a starting point from which more intesive analyses can be done in the future.

\subsection{Quantitatively Classifying Keystone and Redundant Components}

As a means to quantify the relative contribution of elements within functional groups, Davic [31] suggests a variety of community indices could be applied, and uses the dominance index (DI) [60] to determine importance of individual elements within a group:

$$
D I_{B P}=\left(n_{\max } / N\right)
$$

where $n_{\max }$ represents the number of individuals of the most abundant element, and $N$ is the total number of individuals within the functional group as a whole. In cases where more than one potential keystone is identified within a functional group Davic [31] advocates the community dominance index (CDI) of McNaughton [61]:

$$
C D I=\left(n_{1}+n_{2} /\right) N
$$

such that $n_{1}$ and $n_{2}$ represent the frequency of the two most abundant species within a functional group. Other ecological measures of dominance may also provide quantitative insights to the relative importance of individual elements to a group. Commonly applied in the field of ecology is the Simpson Domination Index [62]:

$$
D I_{S}=\sum\left(n_{i} / N\right)^{2}
$$

where $n_{i}$ is the population of each species, and $N$ is the total population. The Simpson method gives greater consideration to diversity within a system; in contrast the Berger-Parker approach [60] does not account for the number of species, but only the total population of the system. However, the Simpson method falls short in that it can only be applied to characterize groups and not individual elements. Our analysis, therefore, applied the Simpson method to the functional groups to provide a more conservative assessment of domination within each group. We then applied the Berger-Parker equation [60] to quantify each species' dominance within the functional groups. Because we applied these indexes to both the groups and their elements, we refer to domination of to characterize the inequality within a group, and to dominance by to describe the contribution of individual elements to a group. For the purposes of this paper, we have determined that a value of $>0.5$ for either $\mathrm{DI}_{\mathrm{BP}}$ or CDI calculations would result in a classification of a biocultural element as a social-ecological keystone, and a value of $<0.5$ would result in a classification of a biocultural element as a social-ecological redundant. 


\section{Results}

A review of the literature regarding Hawaiian agricultural practices [53-59] yielded three classes of crop systems and eighteen biocultural functional groups that span a range of functions, incuding food production, material resource production, and spiritual/religious practice. Dominance by and domination of three sets of cropping systems (Tables 1 and 2) and the eighteen biocultural functional groups (Table 3) was calculated.

Table 1. Dominance Index (DI) as calculated for each of the systems of growing crops in the Hawaiian social-ecological system, as documented by Handy et al. [54].

\begin{tabular}{cccc}
\hline Cropping System & Dominance of Cropping Systems & Domination by Species Assemblage & Associated Crop Species \\
\hline Rain-fed & 0.441 & 0.263 & 11 \\
Agroforestry & 0.288 & 0.110 & 17 \\
Flooded-field & 0.272 & 0.731 & 7 \\
\hline
\end{tabular}

Table 2. Dominance of crops within three major classes of agricultural systems that existed within the Hawaiian social-ecological system.

\begin{tabular}{ccccc}
\hline Latin Name & Hawaiian Name & $\begin{array}{c}\text { Dominance in } \\
\text { Rain-Fed Systems }\end{array}$ & $\begin{array}{c}\text { Dominance in } \\
\text { Agroforestry }\end{array}$ & $\begin{array}{c}\text { Dominance in } \\
\text { Flooded Systems }\end{array}$ \\
\hline Aleurites molaccanus & Kukui & - & $\mathbf{0 . 1 8 6}$ & - \\
Artocarpus altilis & Ulu & - & 0.089 & - \\
Broussonetia papyrifera & Wauke & 0.013 & 0.066 & 0.016 \\
Cocos nucifera & Niu & - & 0.094 & - \\
Colocasia esculenta & Kalo & $\mathbf{0 . 3 5 2}$ & $\mathbf{0 . 1 7 8}$ & $\mathbf{0 . 8 5 2}$ \\
Cordia subcordata & Kou & - & 0.010 & - \\
Cordyline fruticosa & Kō & 0.005 & 0.028 & -014 \\
Curcuma domestica & Ölena & 0.005 & 0.015 & - \\
Dioscorea alata & 'Uhi & 0.073 & 0.036 & - \\
Dioscorea bulbifera & Hoi & - & 0.028 & - \\
Dioscorea pentaphylla & Pi'a & - & 0.008 & - \\
Ipomoea batatas & 'Uala & $\mathbf{0 . 3 4 9}$ & - & - \\
Lageneria siceraria L. vulgaris & Ipu & 0.086 & - & 0.059 \\
Musa ssp. & Mai'a & 0.023 & - \\
Pandanus tectorius & Hala & - & 0.099 & 0.016 \\
Piper methysticum & Awa & 0.040 & 0.084 & - \\
Saccharum offinarum & Kō & 0.043 & 0.008 & - \\
Schizostachyum glaucifolium & Ohe & - & 0.003 & 0.019 \\
Tacca leontopetaloides & Pia & 0.010 & 0.041 & $\mathbf{7}$ \\
\hline Count & & $\mathbf{1 1}$ & 0.028 & $\mathbf{1 7}$ \\
\hline
\end{tabular}

Table 3. The eighteen biocultural functional groups that embody Hawaiian agriculture traditions as identified in the Handy et al. [54] tome on the topic, dominance index (DI) for each, and associated crop species.

\begin{tabular}{|c|c|c|c|c|}
\hline Biocultural Functional Group & $\begin{array}{c}\text { Dominance of Hawaiian } \\
\text { Agriculture }\left(\mathrm{DI}_{\mathrm{S}}\right)\end{array}$ & $\begin{array}{l}\text { Domination of Group } \\
\text { by Crop (DI }\end{array}$ & $\begin{array}{c}\text { Number of } \\
\text { Associated Crops }\end{array}$ & Dominant Crop \\
\hline Complex carbohydrates for food & 0.211 & 0.336 & 9 & Kalo \\
\hline Ceremonial plants for religious practice & 0.108 & 0.277 & 8 & 'Awa \\
\hline Wood (timber, fuel, vessel, music, misc.) & 0.098 & 0.184 & 11 & Kukui/Hau \\
\hline Medicinal applications & 0.069 & 0.105 & 17 & 'Awa \\
\hline Leaves for weaving or thatch material & 0.059 & 0.459 & 4 & Hala \\
\hline Fibers for clothing & 0.037 & 1.000 & 4 & Wauke \\
\hline Simple carbohydrate for food & 0.037 & 0.274 & 6 & Niu \\
\hline Mulch for agriculture & 0.028 & 0.378 & 4 & Kukui \\
\hline
\end{tabular}


Table 3. Cont.

\begin{tabular}{|c|c|c|c|c|}
\hline Biocultural Functional Group & $\begin{array}{c}\text { Dominance of Hawaiian } \\
\left.\text { Agriculture (DI } \text { ( }_{S}\right)\end{array}$ & $\begin{array}{l}\text { Domination of Group } \\
\text { by Crop (DI }\end{array}$ & $\begin{array}{c}\text { Number of } \\
\text { Associated Crops }\end{array}$ & Dominant Crop \\
\hline Oil for culinary uses and healing & 0.026 & 0.501 & 2 & Kukui \\
\hline Genesis story with the culture & 0.020 & 1.000 & 1 & Kalo \\
\hline Leafy greens for food & 0.019 & 0.600 & 3 & Kalo \\
\hline Glue/resin source & 0.002 & 1.000 & 2 & ‘Ulu \\
\hline
\end{tabular}

\section{Analysis}

In examining the dominance of cropping system forms of Hawaiian agriculture (Table 1), 'rain-fed' was the dominant crop system (0.44), followed by agroforestry (0.29), and then flooded-field systems (0.27). However, the split was relatively level, and the Simpson Domination Index $\left(\mathrm{DI}_{\mathrm{S}}\right)$ for Hawaiian Cropping Systems based on these relative abundances yields a moderate value of 0.35 . This indicates that a severe loss of any one of the three systems would substantially impact Hawaiian agriculture. Conversely, the domination of each the three cropping systems by their crop components varied significantly, indicating wildly different levels of reliance on critical species. The agroforestry systems, with a very low value of 0.11 , could easily absorb the loss of any species, including its dominant species. At the opposite end of the spectrum, flooded-field agriculture with an extremely high value of 0.73 would likely catastrophically fail if its most abundant species were to be removed. Rain-fed agriculture, with a more moderate value of 0.26 , would likely struggle but adapt to a removal of the dominant species.

Examining the dominance of crops within these agricultural systems, kalo (taro, Colocasia esculenta) cultivation was either dominant or co-dominant in all three systems (Table 2). Within flooded-field agriculture kalo was highly dominant $\left(\mathrm{DI}_{\mathrm{BP}}=0.85\right)$, a value that classifies it as a keystone component. Within rain-fed systems kalo cultivation was only slightly more dominant (0.352) than 'uala (sweet potato, Ipomoea batatas) cultivation (0.349), indicating clear co-dominance. The CDI value of 0.7 classifies the cultivation of these species as a keystone component, which indicates that rain-fed cultivation would likely collapse without either of the two co-dominant species. Within agroforestry systems kalo cultivation was also co-dominant (0.18) with kukui cultivation (0.19), and was the dominant among non-canopy species. The moderate CDI value of 0.37 suggests that cultivation of either does not play a keystone role, suggesting that agroforestry systems would adapt to the loss of either or both of the co-dominant species.

In examining the eighteen biocultural functional groups (Table 3), cultivating a complex carbohydrate as a food source displayed the highest level of dominance. Other important functional groups $\left(>5 \% \mathrm{DI}_{\mathrm{BP}}\right)$ include religious and ceremonial associations, wood, famine food, thatching, and medicinal uses. Kalo again demonstrates significant importance: it is the dominant species of the dominant functional group, in $43 \%$ of the important functional groups, and in $33 \%$ of all functional groups. Furthermore, it contributes to more functional groups (61\%) than any other species.

An additional finding indicates a relationship between dominance of functional groups within Hawaiian social-ecological system, and how dominated by their species assemblage those functional groups are (Figure 4). This was a highly significant relationship $\left(\mathrm{r}^{2} 0.39, p 0.006\right)$ described by a log-log function $(\log (\mathrm{y})=4.09-0.39 \times \log (\mathrm{x}) ; \operatorname{var}(\mathrm{x})=28.4 ; \operatorname{var}(\mathrm{y})=903.6 ; \operatorname{cov}[\mathrm{x}, \mathrm{y}]=-0.5056)$. Although perhaps intuitive, this indicates that functional groups that make less significant contributions to the social-ecological system are more likely to rely on a smaller assemblage of species. This relationship is important because it indicates that essential functions of a biocultural system will tend to not develop an overly dominant species, likely resulting in increased resilience within the social-ecological system due to functional and responce diversity. In the case of Hawai' $i$, the most dominant functional group-cultivation of complex carbohydrates-has a relatively high $\mathrm{DI}_{S}$ value, indicating a higher reliance on kalo than might be expected for such an important function. 


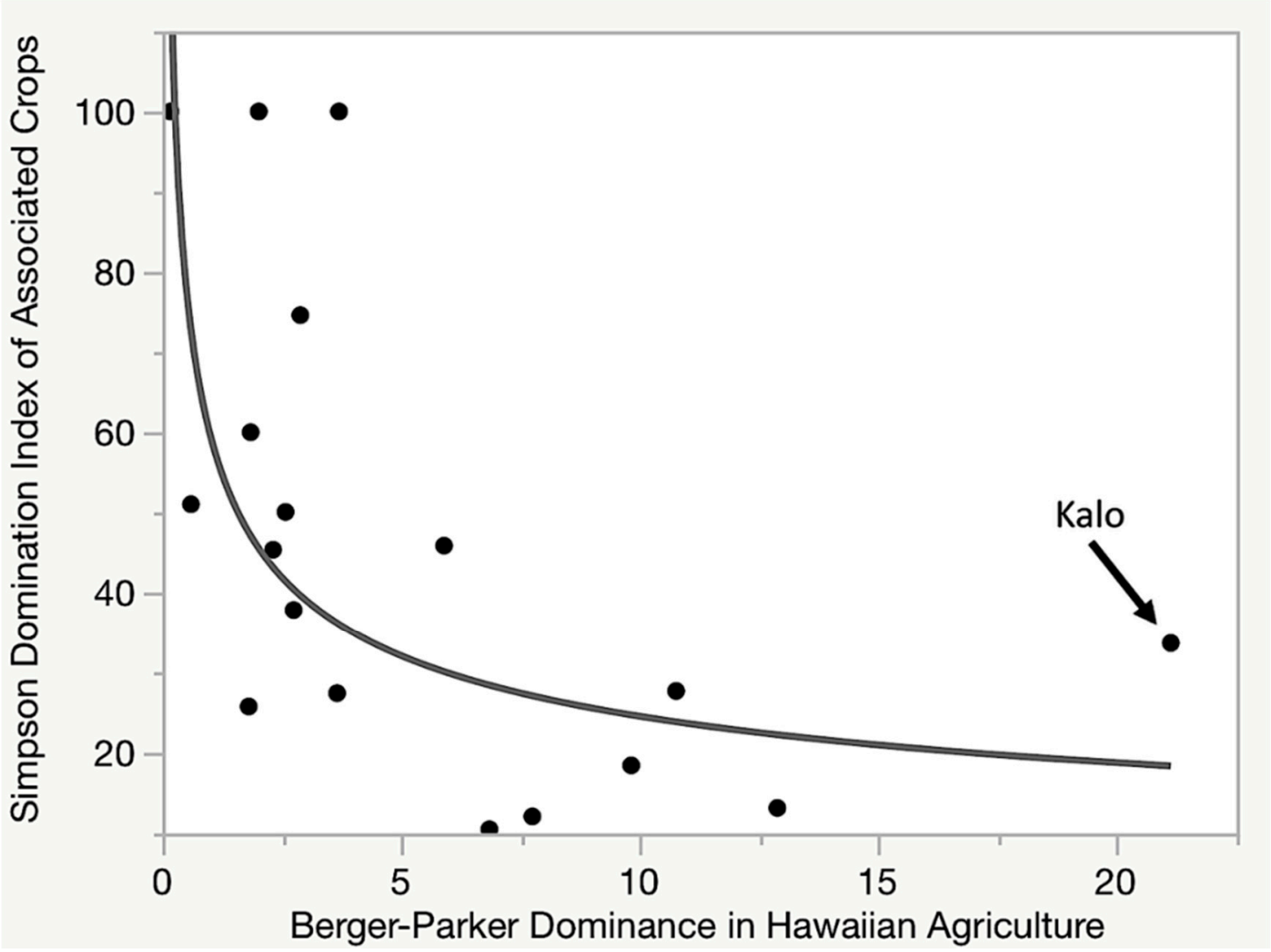

Figure 4. As importance of function groups pertaining to agriculture within the Hawaiian social-ecological systems decreases, the Domination Index within that group tends to increase along a $\log$-log relationship. This indicates the less significant a functional group is, the more heavily it can rely on a smaller species assemblage.

\section{Discussion}

\subsection{Is Kalo Cultivation a Keystone Component of the Hawaiian Social-Ecological System?}

Kalo cultivation displayed high dominance within cropping systems, being dominant or co-dominant in all three systems identified, and within biocultural functional groups, being dominant in multiple important groups. Furthermore, kalo is dominant within the most important functional group-the cultivation of complex carbohydrates. But do the metrics we've identified adequately capture the importance and impacts of kalo within the Hawaiian social-ecological system, especially as relates to structure and function of the system as a whole? These questions are explored in a discussion below.

\subsection{Biocultural Relationships between Kalo and Hawaiian Culture}

Through the original voyages of Polynesians to what is now known as the Hawaiian Islands a total of at least twenty-seven plant species and six animal species were established in Hawai'i [63], collectively referred to as a "portable biocultural toolkit" - a term we apply to the suite of plants and animals that cultural groups take with them in their diaspora across the globe. The taxa selected were likely chosen because they-through their pre-established links to sociocultural practices—could facilitate the perpetuation of key biocultural functional groups (e.g., eating food, drinking liquid, healing with medicine, clothing production, religious ceremony, storing food, etc.) upon arrival at a new destination. They were also the vehicles for transporting stories, which helped retain lessons about life, family, and culturally-appropriate behaviors; and, therefore, represented important teaching tools for future generations. 
As the Hawaiian culture developed, kalo came to hold a pre-eminent role on both a practical and philosophical level. This is reflected in a high level of pre-contact diversity with approximately 400 distinct cultivars of kalo that the Hawaiian culture co-evolved with [18]. Not only was kalo the preferred staple food, but it was also considered to be the original ancestor of the Hawaiian race [54] and the ancient religious system [55,58]. Furthermore, there are many philosophical and symbolic links observed linguistically between the parts and growth form of kalo, and the Hawaiian family system and its structure [54].

Evidence for the central importance at which kalo was deeply ingrained within the identity, dietary system and religious beliefs of the Hawaiian people is seen in the ancient proverb "Ola ke kalo, ola ke kanaka", which roughly translates to "As long as kalo lives, so shall the Hawaiian race"; the converse implication is that if kalo were to disappear, so too would the Hawaiian race [18,64]. It is apparent that due to its central importance in the identity, diet, and religion of the ancient Hawaiian society its hypothetical disappearance would drastically alter the cultural subsystem that was built upon it-not only because of its direct importance, but also because of secondary extinctions which would occur due to the breaking of linked biocultural relationships. Thus, it certainly satisfies the Garibaldi and Turner [11] definition of cultural keystone species, at least within the context of pre-contact Hawaiian culture. One could also argue that the practices associated with kalo satisfy the Brosi et al. [12] definition of "cultural keystone practice" in the same pre-contact period. However, beyond establishing kalo as a "cultural keystone species" and the traditions associated with it as "cultural keystone practices" via an $a$ priori assessment, this paper takes a quantitative approach to determining which specific biocultural relationships can be considered keystone components of the Hawaiian social-ecological system.

\subsection{Kalo Is a Dominant Component in Hawaiian Cropping Systems}

Kalo was a dominant component in all three cropping systems-rain-fed, agroforestry, and flooded-field-employed in the Hawaiian social-ecological system. 'Uala (sweet potato) was co-dominant in rain-fed systems, which is a strong indicator of functional redundancy in that group. This means that the structure of rain-fed systems could, theoretically, still be maintained in such systems, if kalo was not utilized for some reason. The same could not be said for flooded-field systems; the results indicate that kalo's disappearance would result in structural shifts, and potential collapse, in that cropping system. Overall, no cropping system was overbearingly dominant in Hawaiian agriculture when viewed at the scale of the archipelago. However, the spatial distribution of agricultural opportunities was not evenly distributed across the archipelago [27], and the loss of kalo would have had different effects based on the local reliance on different cropping systems. Particularly on the oldest island of Kaua'i, where flooded-field agriculture was highly dominant, the theoretical loss of kalo would likely drastically disrupt the entire society. This would not necessarily be the case in leeward Hawai'i and Maui Islands, where rain-fed agriculture dominated. It could be argued that the functional redundancy was being enacted in rain-fed systems on the younger islands of Maui and Hawai'i, where shifts in the dominant deity (from Kāne to Lono) and the development of religious sects occurred in areas that, due to environmental limitations of kalo cultivation, were dominantly dependent on 'uala.

\subsection{Kalo Is a Dominant in a Key Biocultural Functional Group}

Of all the biocultural functional groups associated with Hawaiian agriculture, the most important was "cultivating a complex carbohydrate as a food source" (Table 3). Within that biocultural functional group, kalo was a strong dominant with no co-dominant (Table 3). Theoretically, this means that if kalo was not used in agriculture systems, for whatever reason, the structure of those systems would shift into an altered state, especially in light of the unique agricultural practices associated with kalo cultivation as described by Handy et al. [54]. In rain-fed systems, kalo could theoretically be replaced by its co-dominant ('uala, sweet potato), but the structure of those systems would shift due to the different agricultural practices associated with the cultivation of each species. For instance, 'uala matures more 
quickly, and also has a more confined harvest and storage time, indicating that compared to kalo, an 'uala-based system would require a more mobile labor force, and more consistent time in the field to manage the shorter harvest periods. More planning and balance would be needed to manage the short window of harvest for constant food supply, and the systems would likely be more vulnerable to both environmental and social perturbances such as drought or war.

In agroforestry systems, kalo's dominance was due to its ability to grow in low light conditions and, more importantly, be able to store in ground for extended periods and self replicate-all traits that are condusive to its cultivation in these systems. It could be replaced by a redundant component of the sub-canopy layer in that system, but the structure of that system would also shift. In flooded-field systems, there was no redundant component in the pre-contact period that could be grown in such flooded conditions. Therefore, if kalo was not cultivated in that system, the lack of a functional redundant would result in an inability of that system to exist, which would result in a structural shift in cropping systems if agriculture was to continue.

\subsection{Cropping Systems Associated with Kalo Influenced the Structure of the Hawaiian Social-Ecological System}

Although not a native plant, kalo played a major role in the highly intensified management of the ecological subsystem, and its cultivation shaped the cultural landscape that is iconically associated with the Hawaiian social-ecological system. Kalo is most productively cultivated in terraced and flooded pond-fields ([54], Figure 5), which resemble rice paddies of South-East Asia. Evidence [27,54,65] suggests that nearly all land capable of being irrigated (from the backs of valleys to the alluvial plains bordering the seashore) was converted from its natural (i.e., pre-human) state of lowland wet-to-mesic forest types; to flooded fields for the cultivation of kalo. This flooded-field system of agriculture shaped the social-ecological system around it as described below.

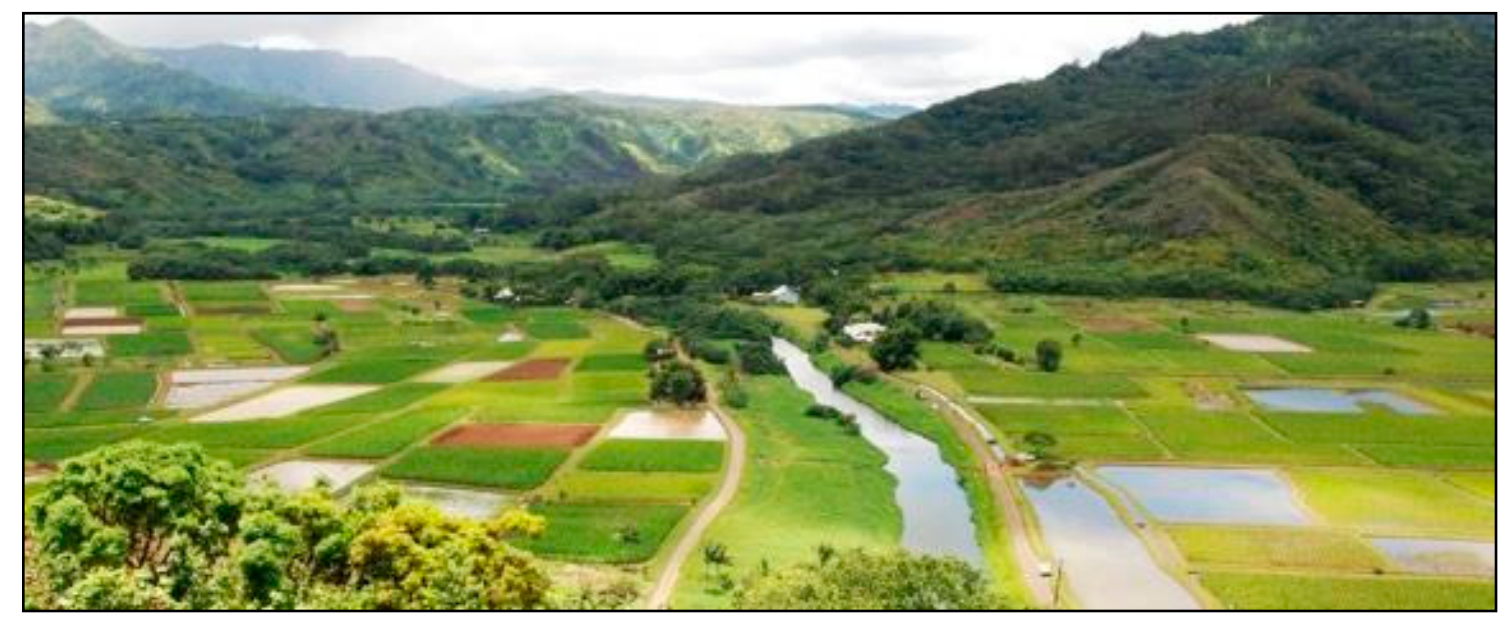

Figure 5. Picture of a contemporary flooded- field system in Hawai'i used to cultivate kalo (taro, Colocasia esculenta). In this style of agricultre, rivers/streams are the central component with its waters being diverted over large areas of adjacent flatlands for the cultivation of kalo.

The conversion of large areas of lowland forest into flooded-field systems had three major repercussions which directly influenced the structure and function of the Hawaiian social-ecological system, as well as the outward appearance of its associated cultural landscapes [66]:

1. This conversion induced localized regime shifts in large areas of land (valley floors and alluvial plains) from forest biome to riparian ecotone. This, in essence, expanded and stabilized riparian habitat—a highly productive ecotone-from a relatively limited to a very broad area. Archaeological evidence suggests that such localized regime shifts have occurred [67], and likely extended the range of native water (i.e., riparian) fowl allowing for increases in their populations [68]. 
2. This conversion theoretically increased the capacity of aquifers (i.e., the islands' ability to retain water). The expansive flooded-field system slowed the flow rate of water on its journey towards the sea, and increased surface area of land covered by water. This cumulatively increased the potential for aquifer recharge. Increasing aquifer recharge potentially increases the level of the aquifer, which could result in more artesian springs at higher elevations than previously existed. The appearance of these springs would further increase the potential for lands-at higher elevation-to be converted to flooded terraces.

3. This conversion likely induced localized regime shifts in estuaries and nearshore reefs from predator-dominated to herbivore-dominated. In theory, this may have been achieved through the development of aquaculture technologies. The emergence of such technologies was likely enabled by the flooded-field system, which mobilized nutrients and then transported them to coastal areas. The water passing through this flooded-field system was presumably enriched due to both a direct and indirect increase in organic matter, and anaerobic soils that mobilized otherwise fixed phosphorous into water systems. This aquaculture system had several classes of fish ponds, including those that walled in large areas of near-shore reef. These walls trapped the enriched water, thus containing algal blooms which allowed for the farming of herbivorous fish within them, while maintaining the health of the reef outside of the walls. This effectively expanded and stabilized estuary habitat—another highly productive ecotone-from a relatively limited to a very broad area. The success of this technology hinged on the management strategies which included methodological removal of top predators.

Each of the above occurrences turned out to be key components to the structure and function of the social-ecological system that existed due to the intensified resource management system of ancient Hawaiian civilization known as the Moku System [25]. The ecosystem services provided likely enabled a population boom in the Hawaiian social-ecological system that was sustained until contact with Europeans in 1778 A.D., which subsequently brought unexpected and catastrophic change such as a $90 \%$ population collapse resulting from introduced diseases [48].

\subsection{Substitution of a Social-Ecological Keystone Alters the Structure of Social-Ecological Systems}

An example of resilience is seen in the functional group "crop grown in rain-fed field systems" (Table 2), which has 'uala as a co-dominant with kalo. Rain-fed cropping systems existed in regions in the Hawaiian archipelago that lacked suffient water for large-scale kalo cultivation (either through rain or surface water). Areas such as this-as in the moku (districts) of Kohala Hema, Kona Akau and Kona Hema on the island of Hawai' $\mathrm{i}$-had field systems which were shaped around 'uala, the co-dominant in rain-fed systems. Lincoln et al. [39] point out that kalo was succeeded by 'uala in regions where kalo could not be cultivated on a large scale due to insufficient water availability. In other words, the dominant of a key biocultural functional group (i.e., the keystone of that biocultural functional group) was substituted by one of the redundant components in that functional group.

Theoretically, this would result in a structural shift within the system, and would look different from the one that was shaped around the cultivation of kalo in flooded-field systems. Indeed, we argue that an "alternative regime state" existed in different regions within the Hawaiian social-ecological system where "uala became dominant. An "alternative regime state" exists when one keystone is succeeded by another in the context of the same social-ecological system. Contemporary analysis of archaeological and historical evidence $[27,39,69]$ supports the notion that this has occurred, and indicates that 'uala cultivation was the keystone component of social-ecological systems in the Hawaiian islands in regions lacking sufficient water for intensified cultivation of kalo. In this case, manifestations were seen in the emergence of religious sects where a shift in primary deities that elevated Lono over Kane and the emergence of new rituals and traditions, such as the makahiki festival that originated on the leeward side of Hawai'i Island. Further differences have been evidenced in the stability of the political hierarchy and the propensity for predatory warfare. 
While these large-scale differences of the structure of the social-ecological system in places where it was shaped around 'uala —as opposed to kalo—are observable, a detailed investigation would likely reveal many more subtle changes at the local level. Kalo expresses extreme dominance in key functional groups such as relation to the family system and the genesis of mankind. How did the local biocultural relationships evolve in the absence of kalo? The redundancy within the agricultural functional groups allowed for the existence of the Hawaiian social-ecological system in arid regions, albeit one of altered form and structure. In ecological analogy, an "alternative regime state" would be akin to replacing the dominant canopy tree in a forest, with cascading effects on the assemblage of bird, animal and insect species present, while a "regime shift" might be shifting from a forested ecosystem to a shrubland, and all the associated shifts in supported species. Similar regime shifts have also been explored by Scheffer et al. [70].

\section{Conclusions}

\subsection{On Keystone and Redundant Components within Social-Ecological Systems}

Social-ecological systems are composed of linked biological-sociocultural relationships, and can be referred to as the "biocultural elements" of these systems. "Keystone" components and "redundant" components exist within the set of biocultural elements that compose social-ecological systems. Theory and methods exist with which to identify and quantify these components, and to correlate them to system health (i.e., system function and resilience), as well as how system health changes over time. Using functional groups to classify and distinguish between keystone components and redundant components is a viable methodology. Such an approach is useful to consider in the context of biocultural restoration of social-ecological systems, as data produced by this approach could be used to influence resource management policies. In accordance with systems theory, this approach could also be applied to other systems-both historical and modern.

However, as in ecology, there are no clearly defined thresholds used to classify components as either a keystone or a redundant, but rather a holistic view of the functional roles must be considered. While quantifying these metics provides insights into the importance of biocultural elements within a social-ecological system, there are no hard cut-off values, or even well-established guidelines, for interpreting data. Therefore, quantification alone cannot define either keystone or redundant components, but qualified assessments can help to illuminate such designations.

Through both quantitative and qualitative methods, we explored functional roles in the Hawaiian social-ecological system, and conclude that kalo qualifies as a keystone species for the Hawaiian culture, and further that kalo cultivation can be considered a keystone component of the Hawaiian social-ecological system. This suggests kalo and its cultivation is vital for the structure and function of the Hawaiian social-ecological system, and that the removal of kalo from parts of this system would result in either alternative regime states, or a regime shift resulting in an entirely new social-ecological system. Historical trends over the last two centuries support this notion.

\subsection{On Biocultural Diversity and Resilience in Social-Ecological Systems}

A loss of biocultural functional groups-due to a lack of both functional redundancy and functional diversity-could induce cascading extinctions on both sides of the social-ecological system. Therefore, increasing biocultural diversity is the most pragmatic way to manage resilience social-ecological systems. Redundancy within biocultural functional groups allows for resilience because of the presence of response diversity in such functional groups. In regions where a particular social-ecological keystone cannot exist, for whatever reason, redundancy in its biocultural functional group can facilitate the existence of an "alternative regime state" within the same social-ecological system, which is built around a successional keystone component. An "alternative regime state" is an altered stable state that is built upon a successional component of a biocultural functional group, one that would likely have a different level of resilience. Such a pehnomenon would also likely result in a cultural landscapes with a different outward appearance occurring within the same social-ecological system. The redundant compontents of Hawaiian agriculture 
made for a resilient social-ecological system. However, a whole-scale removal of these components would theoretically induce a regime shift and result in an entirely new social-ecological system, and this has been historically observed in Hawai'i.

\subsection{On the Model of the Hawaiian Social-Ecological System}

Islands are ideal models for system study because they possess all of the biological and physical processes needed to understand complete systems, yet they exist at a scale where complexity is comprehendible. A relatively good understanding of the Hawaiian social-ecological system exists due to a large body of research conducted by native Hawaiians over the last two centuries, and the existence of well-established research institutions. This makes reasonable speculations about structure and function of this system plausible. While rigorous examination of the original Hawaiian social-ecological system can be challenging — as it is largely associated with an era in the past—due to the large body of information associated with it, creative methodologies can be employed to give insights into its structure and function.

\subsection{Biocultural Restoration of the Hawaiian Social-Ecological System}

In the post-contact era (1778 A.D. onward) Hawaiian culture has been under severe sociocultural pressures such as changes in government, land tenure, religious institutions, economies, language, and others. As a result of these processes native Hawaiians are no longer functioning as the top-level managers of the large-scale social-ecological system that currently exist in Hawai' $i$. These processes and events are analogous to disturbance, uncertainty, and surprise events [35,43]. Despite these potentially catastrophic events, nearly all aspects of the Hawaiian social-ecological system persist into contemporary times. This may be due, at least in part, to the resilience of key biocultural functional groups associated with Hawaiian culture, particularly those which were shaped around flooded-field system agriculture for the production of kalo, waterfowl, and fish. This supports the notion that flooded-field kalo cultivation is the foundation of a Hawaiian cultural landscape; and is, therefore, the key to the biocultural restoration of the Hawaiian social-ecological system. Provided that cultural landscapes are the outward appearance of social-ecological systems, focusing on keystone and redundant elements found within a culture's "portable biocultural toolkit" may provide a pathway for maintaining and/or restoring cultural landscapes. We content that while the pre-colonial state cannot be re-created exactly, by looking to the past we can understand and re-create productive and resilient cultural landscapes. Such cultural restoration goes hand-in-hand with ecological restoration.

\subsection{Future Research}

The theories explored in this paper are not new, but this manuscript puts forth some novel applications of them in the context of social-ecological systems. While the methods presented herein demonstrate some level of credibility to this approach, these notions would need to be assessed in other ways to further test their validity. Some possibilities for future research could include:

- Assessing the percentage of total land area associated with each biocultural functional group to classify between keystone, dominant, and redundant components within social-ecological systems.

- Exploring the functional groups relating to animal husbandry, and assessing dominance in the context of functional groups.

- Expanding these methods to the entire biocultural resource spectrum of a social-ecological unit, which in the Hawaiian archipelago extends from the mountains to sea.

- Assessing the viability of utilizing social-ecological keystones to induce a regime shift back towards the state of abundance known in the Hawaiian language as, "äina momona" or biocultural resource abundance.

Until more rigorous testing can be done, the concepts explored in this manuscript should still be considered theoretical at best. 
Author Contributions: Conceptualization, K.B.W.; Methodology, K.B.W. and N.K.L.; Validation, N.K.L, and F.B.; Formal Analysis, K.B.W., and N.K.L; Investigation, K.B.W., and N.K.L.; Resources, K.B.W.; Data Curation, K.B.W., and N.K.L; Writing-Original Draft Preparation, K.B.W.; Writing-Review \& Editing, K.B.W., N.K.L, and F.B.; Visualization, K.B.W., and N.K.L.; Supervision, F.B.; Project Administration, K.B.W.; Funding Acquisition, K.B.W.

Funding: This research received no external funding. The APC was funded by Hawai'i Community Foundation.

Acknowledgments: We express our gratitude to Will McClatchey, Peter Vitousek, Mary Power, and Kim Bridges who have contributed to our research by providing direction for this paper; and to Joshua Diem, who provided the image used in Figure 3. We also thank National Tropical Botanical Garden, and Department of Tropical Plant and Soil Sciences (University of Hawai'i at Mānoa) for supporting this research.

Conflicts of Interest: The authors declare no competing interests as defined by Sustainability, or other interests that might be perceived to influence the results and/or discussion reported in this paper. The founding sponsors had no role in the design of the study; in the collection, analyses, or interpretation of data; in the writing of the manuscript and in the decision to publish the results.

\section{References}

1. Balée, W. The research program of historical ecology. Annu. Rev. Anthropol. 2006, 35, 75-98. [CrossRef]

2. Berkes, F.; Colding, J.; Folke, C. (Eds.) Navigating Social-Ecological Systems: Building Resilience for Complexity and Change; Cambridge University Press: Cambridge, UK, 2003.

3. Berkes, F.; Folke, C. (Eds.) Linking Social and Ecological Systems: Management Practices and Social Mechanisms for Building Resilience; Cambridge University Press: Cambridge, UK, 1998.

4. Ostrom, E. A General Framework for Analyzing Sustainability of Social-Ecological Systems. Science 2009, 325, 419-422. [CrossRef] [PubMed]

5. McGinnis, M.D.; Ostrom, E. Social-ecological system framework: Initial changes and continuing challenges. Ecol. Soc. 2014, 19, 30. [CrossRef]

6. Pieter, B.; Schlüter, M.; Sendzimir, J. A Framework for Analyzing, Comparing, and Diagnosing Social-Ecological Systems. Ecol. Soc. 2015, 20, 18.

7. Sterling, E.J.; Filardi, C.; Newell, J.; Albert, S.; Alvira, D.; Bergamini, N.; Betley, E.; Blair, M.; Boseto, D.; Burrows, K.; et al. Biocultural approaches to sustainability indicators: Bridging local and global scales to foster human adaptive capacity and ecological resilience. Nat. Ecol. Evol. 2017. [CrossRef]

8. Walker, B.; Holling, C.S.; Carpenter, S.R.; Kinzig, A. Resilience, adaptability and transformability in social-ecological systems. Ecol. Soc. 2004, 9, 5. [CrossRef]

9. Berkes, F. Restoring Unity: The Concept of Marine Social-Ecological Systems. In World Fisheries: A Social-Ecological Analysis; Ommer, R.E., Perry, R.I., Cochrane, K., Cury, P., Eds.; Wiley-Blackwell: Oxford, UK, 2011.

10. Cristancho, S.; Vining, J. Culturally defined keystone species. Hum. Ecol. Rev. 2004, 11, 153-162.

11. Garibaldi, A.; Turner, N. Cultural keystone species: Implications for ecological conservation and restoration. Ecol. Soc. 2004, 9, 1. [CrossRef]

12. Brosi, B.J.; Balick, M.; Wolkow, R.; Lee, R.; Kostka, M.; Raynor, W.; Gallen, R.; Raynor, A.; Lee, L.D. Quantifying cultural erosion and its relationship to biodiversity conservation: Canoe-making knowledge in Pohnpei, Micronesia. Conserv. Biol. 2007, 21, 875-879. [CrossRef] [PubMed]

13. Nguyen, M.L.T.; Wieting, J.; Doherty, K.T. Vegetation analysis of urban ethnic markets shows supermarket generalists and Chinatown ethnic-specialist vendors. Ethnobot. Res. Appl. 2008, 6, 63-85. [CrossRef]

14. Anderies, J.M.; Janssen, M.A. Sustaining the Commons; Center for the Study of Institutional Diversity, Arizona State University: Tempe, AZ, USA, 2013.

15. Norgaard, R.B. Sociosystem and ecosystem coevolution in the Amazon. J. Environ. Econ. Manag. 1981, 8, 238-254. [CrossRef]

16. Winter, K.; McClatchey, W. Quantifying Evolution of Cultural Interactions with Plants: Implications for Managing Diversity for Resilience in Social-Ecological System. In Ethnobotany: A Focus on Brazil. Functional Ecosystems and Communities; Albuquerque, U.P., Ed.; Global Science Books Ltd.: Isleworth, UK; Ikenobe, Japan, 2008; Volume 2, pp. 1-10.

17. Winter, K.B.; McClatchey, W. The Quantum Co-evolution Unit: An Example of Awa (Piper methysticum G. Foster) in Hawaiian Culture. Econ. Bot. 2009, 63, 353-362. [CrossRef]

18. Winter, K.B. Kalo [Hawaiian Kalo: Colocasia esculenta (L.) Schott] Varieties: An Assessment of Ancient and Modern Synonymy and Diversity. Ethnobot. Res. Appl. 2012, 10, 423-447. 
19. Kirch, P.V. Hawaii as a model for human ecodynamics. Am. Anthropol. 2007, 109, 8-26. [CrossRef]

20. Wilmshurst, J.M.; Hunt, T.L.; Lipo, C.P.; Anderson, A.J. High-precision radiocarbon dating shows recent and rapid initial human colonization of East Polynesia. Proc. Natl. Acad. Sci. USA 2011, 108, 1815-1820. [CrossRef] [PubMed]

21. Kamakau, S.M. Ruling Chiefs of Hawaii; Kamehameha Schools Press: Honolulu, HI, USA, 1961.

22. Kame'eleihiwa, L. Native Land and Foreign Desires; Bishop Museum Press: Honolulu, HI, USA, 1992.

23. Cachola-Abad, C.K. Evaluating the orthodox dual settlement model for the Hawaiian Islands: An analysis of artefact distribution and Hawaiian oral traditions. In The Evolution and Organization of Prehistoric Society in Polynesia; Graves, M.W., Green, R.C., Eds.; New Zealand Archaeological Association Monograph: Auckland, New Zealand, 1993; Volume 19, pp. 13-32.

24. Cachola-Abad, C.K. An Analysis of Hawaiian Oral Traditions: Descriptions and Explanations of the Evolution of Hawaiian Socio-Political Complexity. Doctoral Dissertation, University of Hawaii at Mānoa, Honolulu, HI, USA, 2000.

25. Winter, K.B.; Beamer, K.; Vaughan, M.B.; Friedlander, A.M.; Kido, M.; Whitehead, A.N.; Akutagawa, M.K.H.; Kurashima, N.; Lucas, M.P.; Nyberg, B. The Moku System: Managing biocultural resources for abundance within social-ecological regions in Hawai'i. Sustainability 2018, in press.

26. Power, M.E.; Tilman, D.; Estes, J.A.; Menge, B.A.; Bond, W.J.; Mills, L.S.; Daily, G.; Castilla, J.C.; Lubchenco, J.; Paine, R.T. Challenges in the quest for keystones. BioScience 1996, 46, 609-620. [CrossRef]

27. Ladefoged, T.N.; Kirch, P.V.; Gon, S.M.; Chadwick, O.A., III; Hartshorn, A.S.; Vitousek, P.M. Opportunities and constraints for intensive agriculture in the Hawaiian archipelago prior to European contact. J. Archaeol. Sci. 2009, 36, 2374-2383. [CrossRef]

28. Kirch, P.V.; Zimmerer, K.S. Roots of Conflict; School for Advanced Research Press: Santa Fe, NM, USA, 2011.

29. Winter, K.B.; Lucas, M. Spatial modeling of social-ecological management zones of the alii era on the island of Kauai with implications for large-scale biocultural conservation and forest restoration efforts in Hawaii. Pac. Sci. 2017, 71, 457-477. [CrossRef]

30. Paine, R.T. The Pisaster-Tegula Interaction: Prey, Patches, Predator Food Preference, and Intertidal Community Structure. Ecology 1969, 50, 950-961. [CrossRef]

31. Davic, R.D. Linking Keystone Species and Functional Groups: A New Operational Definition of the Keystone Species Concept. Conserv. Ecol. 2003, 7, r11. [CrossRef]

32. Von Bertalanffy, L. General System Theory; George Braziller, Inc.: New York, NY, USA, 1968; Volume 41973, p. 40.

33. Pimm, S.L.; Gilpin, M.E. Theoretical issues in conservation biology. In Perspectives in Ecological Theory; Roughgarden, J., May, R., Levin, S., Eds.; Princeton University Press: Princeton, NJ, USA, 1989; pp. 287-305.

34. Christianou, M.; Ebenman, B. Keystone Species and Vulnerable Species in Ecological Communities: Strong or Weak Interactors? J. Theoret. Biol. 2005, 235, 95-103. [CrossRef] [PubMed]

35. Folke, C.; Carpenter, S.; Walker, B.; Scheffer, M.; Elmqvist, T.; Gunderson, L.; Holling, C.S. Regime shifts, resilience, and biodiversity in ecosystem management. Annu. Rev. Ecol. Evol. Syst. 2004, 35, 557-581. [CrossRef]

36. Rhindos, D. Darwinism and its role in the explanation of domestication. In Foraging and Farming: The Evolution of Plant Exploitation; Harris, D.R., Hillman, G.C., Eds.; Unwin Hyman: London, UK, 1989; pp. $27-41$.

37. Lincoln, N.K.; Vitousek, P.M. Indigenous Polynesian agriculture in Hawaii. Oxford Resear. Encycl. Environ. Sci. 2017. [CrossRef]

38. Kirch, P.V. The Wet and the Dry: Irrigation and Agricultural Intensification in Polynesia; University of Chicago Press: Chicago, IL, USA, 1994.

39. Lincoln, N.K.; Rossen, J.; Vitousek, P.; Kahoonei, J.; Shapiro, D.; Kalawe, K.; Pai, M.; Marshall, K.; Meheula, K. Restoration of 'Āina Malo'o on Hawai'i Island: Expanding Biocultural Relationships. Sustainability 2018, under review.

40. Holling, C.S. Resilience and stability of ecological systems. Annu. Rev. Ecol. Syst. 1973, 4, 1-23. [CrossRef]

41. Resilience Alliance. 2002. Available online: https://www.resilience.org/about-resilience/ (accessed on 30 June 2018).

42. Holling, C.S. Engineering resilience versus ecological resilience. In Engineering within Ecological Constraints; Schulze, P.C., Ed.; National Academy Press: Washington, DC, USA, 1996.

43. Elmqvist, T.; Folke, C.; Nystrom, M.; Peterson, G.; Bengtsson, J.; Walker, B.; Norberg, J. Response Diversity and Ecosystem Resilience. Front. Ecol. Environ. 2003, 1, 488-494. [CrossRef]

44. Naeem, S. Species Redundancy and Ecosystem Reliability. Conserv. Biol. 1998, 12, 39-45. [CrossRef] 
45. Scheffer, M.; Carpenter, S.; Foley, J.A.; Folke, C.; Walker, B. Catastrophic Shifts in Ecosystems. Nature 2001, 413, 591-596. [CrossRef] [PubMed]

46. Fosberg, F.R. (Ed.) Man's Place in Island Ecosystems: A Symposium; Bishop Museum Press: Honolulu, HI, USA, 1962; 264p.

47. Vitousek, P. Nutrient Cycling and Limitation: Hawaii as a Model; Princeton University Press: Princeton, NJ, USA, 2004.

48. Stannard, D.E. Before the Horror: The Population of Hawaii on the Eve of Western Contact; University of Hawaii Press: Honolulu, HI, USA, 1994.

49. Swanson, D.A. The Number of Native Hawaiians and Part Hawaiians in Hawaii, 1778 to 1900: Demographic Estimates by Age, with Discussion; University of California: Riverside, CA, USA, 2015.

50. Hommon, R.J. The Ancient Hawaiian State: Origins of a Political Society; Oxford University Press: Oxford, UK, 2013.

51. Max-Neef, M.; Elizalde, A.; Hopenhayn, M.; Herrera, F.; Zemelman, H.; Jataba, J.; Weinstein, L. Human Scale Development: A Hope for the Future. Dev. Dial. 1989, 1, 5-80.

52. Max-Neef, M.; Elizalde, A.; Hopenhayn, M. Human Scale Development: Conception, Application and Further Reflections; The Apex Press: New York, NY, USA, 1991; 114p.

53. Ii, J.P. Fragments of Hawaiian History; Bishop Museum Press: Honolulu, HI, USA, 1959.

54. Handy, E.S.C.; Handy, E.G.; Pukui, M.K. Native Planters in Old Hawaii: Their Life, Lore, and Environment; Bishop Museum Press: Honolulu, HI, USA, 1972.

55. Kamakau, S.M. Ka Hana a Ka Poe Kahiko; Translated from the Newspaper Ke Au Okoa; Bishop Museum Press: Honolulu, HI, USA, 1976.

56. Kamakau, S.M. Ka Poe Kahiko; Translated from the Newspaper Ke Au Okoa; Bishop Museum Press: Honolulu, HI, USA, 1991.

57. Desha, S.L. Kamehameha and His Warrior Kekuhaupio; Frazier, Frances N., Translator; Kamehameha Schools Press: Honolulu, HI, USA, 2000.

58. Malo, D. Ka Moolelo Hawaii: Hawaiian Traditions; Chun, Malcolm, Translator; First People's Publications: Honolulu, HI, USA, 2006; 274p.

59. Kepelino. Kepelino's Traditions of Hawaii; Beckwith, M.W., Ed.; Bishop Museum Press: Honolulu, HI, USA, 2007; 206p.

60. Berger, W.H.; Parker, F.L. Diversity of planktonic Foraminifera in deep sea sedimanets. Science 1970, 168, 1345-1347. [CrossRef] [PubMed]

61. McNaughton, S.J. Structure and Function in California Grasslands. Ecology 1968, 49, 962-972. [CrossRef]

62. Simpson, E.H. Measurement of diversity. Nature 1949, 163, 688. [CrossRef]

63. Abbott, I.A. Lāau Hawaii Traditional Hawaiian Uses of Plants; Bishop Museum Press: Honolulu, HI, USA, 1992.

64. Kelly, M. Ahupua'a Fishponds and Loi: A Film for Our Time; Nā Maka o ka Āina: Honolulu, HI, USA, 1992.

65. Handy, E.S.C. The Hawaiian Planter: His Plants, Methods and Areas of Cultivation; Bishop Museum Press: Honolulu, HI, USA, 1940; 227p.

66. Molnar, Z.; Berkes, F. Role of traditional ecological knowledge in linking cultural and natural capital in cultural landscapes. In Reconnecting Natural and Cultural Capital: Contributions from Science and Policy; Paracchini, M.L., Zingari, P.C., Blasi, C., Eds.; European Union: Luxembourg, 2018; pp. 183-193.

67. Burney, D.A.; Kikuchi, W. A millennium of human activity at Makauwahi Cave, Mahaulepu, Kauai. Hum. Ecol. 2006, 34, 219-247. [CrossRef]

68. Burney, D.A.; James, H.F.; Burney, L.P.; Olson, S.L.; Kikuchi, W.; Wagner, W.L.; Burney, M.; McCloskey, D.; Kikuchi, D.; Grady, F.V.; et al. Fossil evidence from a diverse biota from Kauai and its transformation since human arrival. Ecol. Monogr. 2001, 71, 615-641.

69. Kurashima, N.; Jeremiah, J.; Ticktin, T. I ka wa ma mua: The value of a historical ecology approach to ecological restoration in Hawaii. Pac. Sci. 2017, 71, 437-456. [CrossRef]

70. Scheffer, M.; Carpenter, S.R.; Lenton, T.M.; Bascompte, J.; Brock, W.; Dakos, V.; Van de Koppel, J.; Van de Leemput, I.A.; Levin, S.A.; Van Nes, E.H.; et al. Anticipating critical transitions. Science 2012, 388, 344-348. [CrossRef] [PubMed]

(C) 2018 by the authors. Licensee MDPI, Basel, Switzerland. This article is an open access article distributed under the terms and conditions of the Creative Commons Attribution (CC BY) license (http://creativecommons.org/licenses/by/4.0/). 